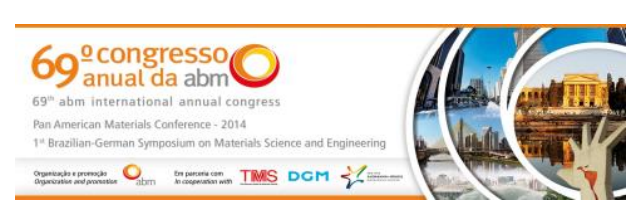

Tema: Lavra e Tratamento de Minérios

\title{
ADSORÇÃO DE DODECILAMINA E OLEATO DE SÓDIO EM SUPERFÍCIES MINERAIS*
}

\section{Resumo}

Daniel Geraldo da Cruz ${ }^{1}$ Daniela Moreira de Oliveira² Diogo Rodrigues Prado ${ }^{3}$

Fábio de São José4

Otávia Martins Silva Rodrigues 5

Medidas de adsorção, tensão superficial e potencial zeta (PZ) são as ferramentas mais usadas para entender os fenômenos interfaciais e, consequentemente, os aspectos químicos envolvidos nos processos de flotação. No presente trabalho avaliou-se a adsorção dos coletores oleato de sódio (OLS) e dodecilamina (DDA) em hematita e quartzo, respectivamente, a partir da medida indireta da adsorção dos reagentes baseada em determinações das tensões superficiais das soluções residuais dos coletores após adsorção. Mediu-se também o potencial zeta dos minerais estudados em função da concentração dos coletores. As medidas indicaram que ocorreu a adsorção. Em relação à hematita os resultados sugerem a ocorrência de adsorção química. Para os ensaios realizados variando-se o tempo de adsorção, observou-se o aumento na adsorção de OLS sobre a hematita em função do tempo. Para o sistema quartzo-dodecilamina os resultados não apresentaram tendência quanto à influência do tempo na adsorção. Esse comportamento sugere que a cinética de adsorção de dodecilamina sobre o quartzo é mais rápida, ocorrendo adsorção nos primeiros instantes de contato entre a solução e o mineral.

Palavras-chave: Adsorção; Tensão Superficial; Potencial Zeta; Coletores.

\section{ADSORPTION OF DODECYLAMINE AND SODIUM OLEATE ON MINERALS SURFACES Abstract}

The interfacial phenomena are closely related to flotation. Adsorption measurements, surface tension and zeta potential are the tools most commonly used to understand these phenomena, therefore, the chemical aspects involved in flotation processes. This study evaluated the adsorption of collectors sodium oleate and dodecylamine in hematite and quartz, respectively. Indirect adsorption measurements of reagents were based on measurements of surface tension of solutions of collectors used. Besides, were done measurements of zeta potential of the mineral studied as function of the concentration of collectors. Hematite results suggest the occurrence of chemical adsorption. The increase in adsorption time resulted in increased of adsorption of oleate in the hematite, this tendency was not observed for the system quartzdodecylamine. The results suggest that the adsorption kinetics is very fast, occurring adsorption in the first moments of contact between the solution containing the collector and the mineral quartz.

Keywords: Adsorption; Surface tension; Zeta potential; Collectors.

1 Engenheiro Metalúrgico, Aluno de Pós-Graduação (Mestrado), Programa de Pós-Graduação em Engenharia Mineral, Departamento de Engenharia de Minas, Universidade Federal de Ouro Preto (UFOP), Ouro Preto, Minas Gerais, Brasil.

2 Engenheira Geológica, Aluna de Pós-Graduação (Mestrado), Programa de Pós-Graduação em Engenharia Mineral, Departamento de Engenharia de Minas, UFOP, Ouro Preto, Minas Gerais, Brasil.

3 Engenheiro de Controle e Automação, Aluno de Pós-Graduação (Mestrado), Programa de PósGraduação em Engenharia Mineral, Departamento de Engenharia de Minas, UFOP, Ouro Preto, Minas Gerais, Brasil.

4 Engenheiro Metalúrgico, Aluno de Pós-Graduação (Mestrado), Programa de Pós-Graduação em Engenharia Mineral, Departamento de Engenharia de Minas, UFOP, Ouro Preto, Minas Gerais, Brasil.

5 Professora. Departamento de Engenharia de Minas, UFOP, Ouro Preto, Minas Gerais, Brasil.

\footnotetext{
* Contribuição técnica ao 69 Congresso Anual da ABM - Internacional e ao 14ํㅡㄹ ENEMET - Encontro Nacional de Estudantes de Engenharia Metalúrgica, de Materiais e de Minas,21 a 25 de julho de 2014, São Paulo, SP, Brasil.
} 


\section{INTRODUÇÃO}

A flotação é um método bastante utilizado para a separação do mineral minério da ganga, seja na forma direta, flotando o mineral minério, ou na forma reversa flotando a ganga. Para conseguir essa seletividade a flotação explora as diferenças de propriedades de superfícies entre os minerais presentes [1].

Para se entender melhor as características da flotação é preciso conhecer os fenômenos de superfície envolvidos, como adsorção, potencial zeta e tensão superficial, pois esses fenômenos estão intimamente ligados com a interação mineral/coletor. Oko [2] afirmou em sua tese, que o objetivo dos estudos dos fenômenos interfaciais era na tentativa de entender melhor o processo de flotação. Kursun e Ates [3] afirmaram que há relação entre a adsorção de coletores e recuperação em uma coluna flotação, o que só comprova que os fenômenos de superfícies devem ser entendidos mais profundamente para o entendimento da flotação.

A flotação da hematita é realizada utilizando coletores aniônicos, um coletor muito utilizado nos estudos é o oleato de sódio. Assim, foram realizadas várias análises na tentativa de determinar quais são as melhores condições para a flotação direta da hematita utilizando oleato de sódio, como pH e concentração do reagente, alguns desses tentando entender primeiro os fenômenos interfaciais da reação partícula/reagente.

Nascimento [4] realizou estudos de flotação de hematita utilizando oleato de sódio como coletor, e chegou a conclusão que o melhor pH para flotação foi entre 7 e 9. Lopes e Lima [5]e Kulkarni e Somasundaran [6] inicialmente determinaram o potencial zeta do mineral na tentativa de encontrar uma relação com a flotabilidade. Nessa análise o PCZ encontrado para a hematita foi 7,5 e 7,1, respectivamente. Nesse mesmo estudo, Lopes e Lima [5], analisando o potencial zeta após a adsorção, perceberam uma queda pouco acentuada no módulo do PZna para a faixa de $\mathrm{pH}$ entre 2 e 4, que indica a presença de adsorção eletrostática, uma queda brusca em pH maior que 7 indicando adsorção química.Shibata e Fuerstenau [7] analisaram a variação da adsorção e do potencial zeta com a variação da concentração do oleato de sódio e notaram grandes diferenças nas curvas, também analisaram as variações de recuperação na flotação variando o pH.

Observando as alterações de características superficiais com a variação de algumas variáveis, é claro que os fenômenos precisam ser mais bem entendidos, principalmente a adsorção do coletor, pois essa adsorção tem como objetivo tornar a partícula hidrofóbica promovendo a flotação.

A flotação de quartzo tradicionalmente é feita com o uso de alguma das variedades de amina como reagente coletor e a forma primária do reagente pode ser obtida a partir de amônia na qual ocorre substituição de um átomo de hidrogênio por um grupo orgânico, levando a formação de cadeias com 8 a 10 carbonos. A partir da amina primária, outros coletores catiônicos foram desenvolvidos e têm sido frequentemente utilizados, a exemplo da dodecilamina na flotação de minério de ferro.

Estudos de adsorção de misturas de coletores em silicatos realizados por Viana et al., [8], usando somente dodecilamina na concentração de $3,5 \times 10^{-5} \mathrm{~mol} / \mathrm{L}$, conferiram ao quartzo, à microclina e à muscovita flotabilidades de $30 \%, 60 \%$ e $35 \%$, respectivamente. Já o uso do dodecilsulfonato, um coletor aniônico, aumenta a flotabilidade da microclina para $80 \%$ mas, praticamente, não altera a flotabilidade do quartzo, que alcança $32 \%$.

\footnotetext{
* Contribuição técnica ao 69 Congresso Anual da ABM - Internacional e ao 14ํㅡㄹ ENEMET - Encontro Nacional de Estudantes de Engenharia Metalúrgica, de Materiais e de Minas,21 a 25 de julho de 2014, São Paulo, SP, Brasil.
} 


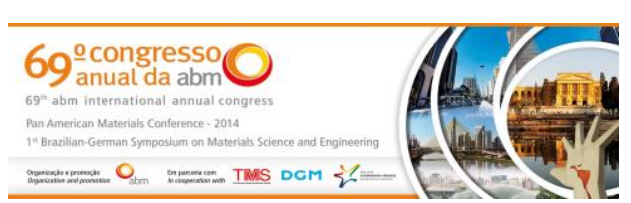

As aminas se adsorvem fisicamente na superfície do quartzo predominantemente por atração eletrostática, pois a superfície do mineral possui quantidade negativa de carga em valores de $\mathrm{pH}$ acima do PIE. O ponto isoelétrico do quartzo situa-se na faixa de $\mathrm{pH}$ entre 2 e 4 possibilitando a adsorção em uma ampla faixa de $\mathrm{pH}$ [9].

Uma adsorção física é caracterizada por uma baixa energia de adsorção e pouca seletividade já que, a princípio, qualquer superfície com carga contrária é atraente para a molécula do coletor. A baixa energia envolvida na interação permite, por exemplo, a reciclagem do reagente [10].

Sendo assim, esse trabalho objetivou a melhor compreensão da adsorção de coletores em superfícies minerais. No presente trabalho avaliou-se a adsorção dos coletores oleato de sódio (OLS) e dodecilamina (DDA) em hematita e quartzo, respectivamente.

\section{MATERIAIS E MÉTODOS}

As quantificações da adsorção dos coletores foram inferidas de maneira indireta, a partir de medidas de tensões superficiais das soluções residuais dos coletores após adsorção. Mediu-se também o potencial zeta dos minerais estudados em função da concentração dos coletores.

Foram utilizadas amostras minerais com granulometria inferior a $75 \mu \mathrm{m}$ para as medidas de potencial zeta e granulometria na faixa $-295+37 \mu \mathrm{m}$ para os testes de adsorção.

As concentrações das soluções de DDA e OLS (solução mãe ou padrão) foram, respectivamente, $500 \mathrm{mg} / \mathrm{L}$ e $1000 \mathrm{mg} / \mathrm{L}$. As demais concentrações utilizadas nos ensaios foram preparadas a partir das soluções padrão.

Para controle de $\mathrm{pH}$ foram utilizadas soluções de $\mathrm{HCl}$ e $\mathrm{NaOH}$. As amostras utilizadas nos testes de adsorção também foram caracterizadas em termos de área superficial específica.

\subsection{Tensão Superficial}

As medidas de tensão superficial foram realizadas utilizando o método do anel, em um tensiômetro modelo K6 do fabricante Krüss, disponível no laboratório do Departamento de Engenharia de Minas da Universidade Federal de Ouro Preto. As medidas foram realizadas em triplicata.

\subsection{Potencial Zeta}

Na determinação do potencial zeta utilizou-se o aparelho modelo Zeta-Meter 4.0. Em uma solução formada por OLS com um eletrólito indiferente $\left(\mathrm{NaCl} 10^{-3} \mathrm{~mol} / \mathrm{L}\right)$, adicionou-se 50mg de hematita pura.

Procedeu-se igualmente com o quartzo, porém com uma massa de $100 \mathrm{mg}$. Ajustouse 0 pH igual a 8,0 da solução resultante, para cada medida de potencial zeta. Após cinco minutos de sedimentação, recolheu-se parte da suspensão para adição na célula eletroforética e a medição do potencial.

\footnotetext{
* Contribuição técnica ao 69ำ Congresso Anual da ABM - Internacional e ao 14ํㅡㄹ ENEMET - Encontro Nacional de Estudantes de Engenharia Metalúrgica, de Materiais e de Minas,21 a 25 de julho de 2014, São Paulo, SP, Brasil.
} 


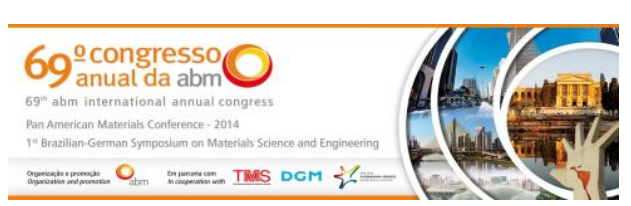

\subsection{Medidas de Adsorção}

\subsubsection{Influência da massa de mineral}

Para estimar a influência da massa de mineral na adsorção do coletor, definiram-se três massas: $2 \mathrm{~g}, 5 \mathrm{~g}$ e $10 \mathrm{~g}$. Em um béquer de $100 \mathrm{~mL}$ juntou-se a massa mineral com $50 \mathrm{~mL}$ da solução de seu respectivo coletor na concentração micelar crítica, CMC, definida no ensaio de tensão superficial.

Posteriormente fez-se a agitação da composição com auxílio de um agitador magnético por trinta minutos para propiciar 0 fenômeno da adsorção. Sequencialmente deu-se um prazo de dez minutos para permitir a decantação do excesso de mineral em suspensão. Finalmente, mediu-se a tensão superficial do sobrenadante recolhido por uma pipeta tomando-se o cuidado de extrair somente a solução a meia altura do béquer.

\subsubsection{Influência do tempo de adsorção}

A cinética da adsorção foi investigada variando o intervalo de tempo no qual uma massa mineral de $5 \mathrm{~g}$ ficou em contato com a solução de coletor. Dessa forma, foram definidos 0,$5 ; 2 ; 15 ; 30 ; 60$ e 120 minutos para agitação da solução de coletor $(50 \mathrm{~mL})$ com os minerais. Em seguida, assim como no item 2.3.1, procedeu-se a medida da tensão superficial da solução residual para estimar, indiretamente, a quantidade de coletor adsorvida.

\section{RESULTADOS E DISCUSSÃO}

A análise de área superficial específica das amostras minerais resultaram em $3,39 \mathrm{~m}^{2} / \mathrm{g}$ para a hematita e $0,13 \mathrm{~m}^{2} / \mathrm{g}$ para o quartzo.

\subsection{Tensão superficial das soluções de DDA e de OLS}

As medidas da tensão superficial em função da concentração da solução de DDA e de OLS estão apresentadas na Tabela 1. A temperatura ambiente verificada durante a realização dos ensaios foi $21^{\circ} \mathrm{C}$ e a concentração zero refere-se à água pura.

Tabela 1.Tensão superficial média em função da concentração da solução de DDA e OLS

\begin{tabular}{ccc}
\hline Concentração [mg/L] & Tensão Superficial [mN/m] \\
& DDA & OLS \\
\hline 0 & 73,2 & 73,1 \\
\hline 2 & 68,8 & 57,2 \\
\hline 10 & 55,2 & 47,2 \\
\hline 20 & 53,5 & 41,7 \\
\hline 50 & 48,0 & 37,1 \\
\hline 100 & 31,5 & 32,9 \\
\hline 200 & - & 28,6 \\
\hline 300 & 27,5 & 28,3 \\
\hline 500 & 27,5 & 28,2 \\
\hline 1000 & - & 28,5 \\
\hline
\end{tabular}

A Figura 1 mostra o efeito do aumento da concentração de DDA e OLS sobre a tensão superficial das soluções em diferentes concentrações.

* Contribuição técnica ao 69ำ Congresso Anual da ABM - Internacional e ao 14ํㅡㄹ ENEMET - Encontro Nacional de Estudantes de Engenharia Metalúrgica, de Materiais e de Minas,21 a 25 de julho de 2014, São Paulo, SP, Brasil. 

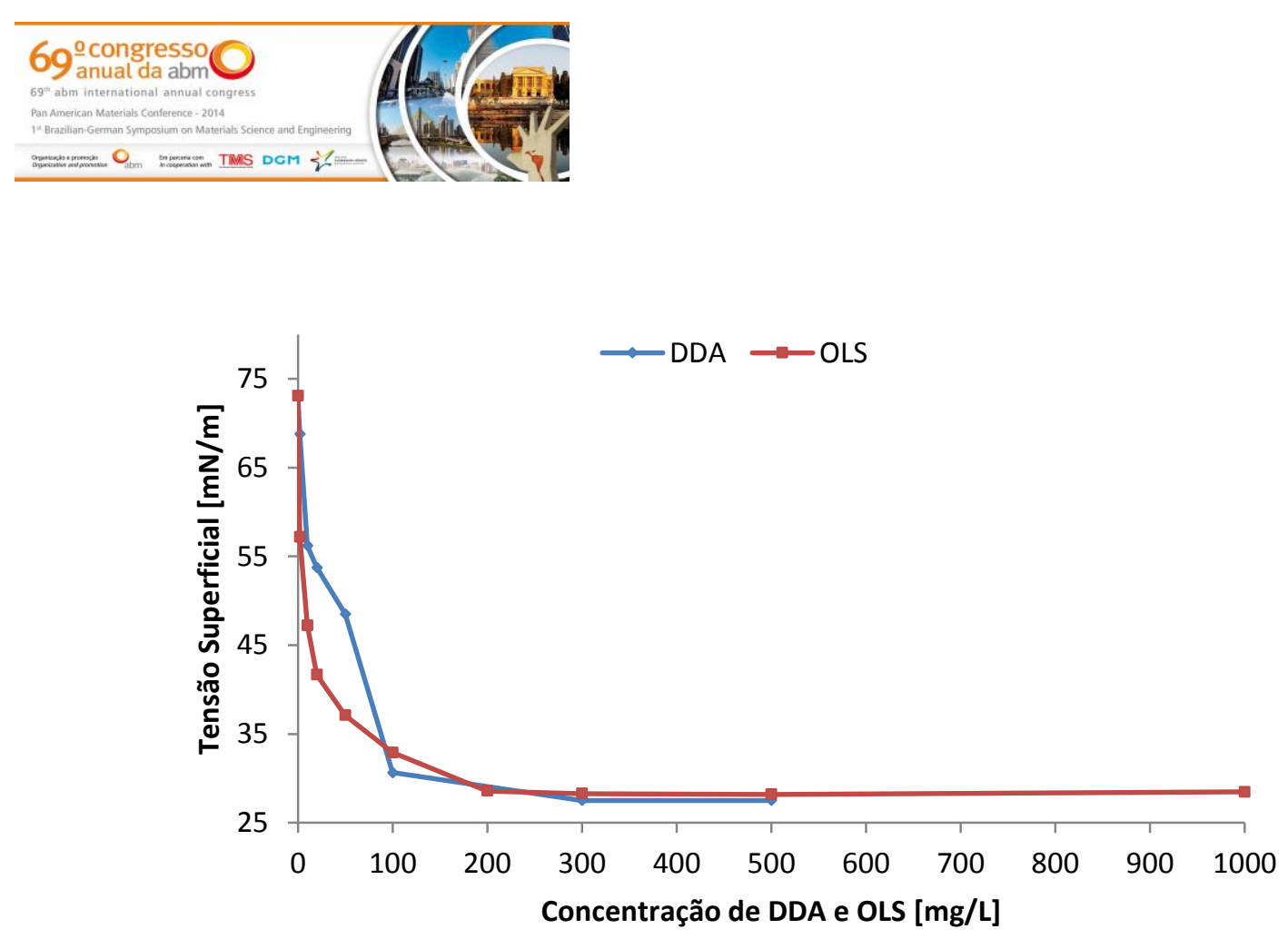

Figura 1.Tensão superficial para diferentes concentrações de DDA e OLS.

Analisando o comportamento das curvas, percebe-se que o aumento da concentração do reagente provoca uma diminuição nos valores da tensão superficial da solução, até alcançar a CMC (concentração micelar crítica), a partir da qual um aumento na concentração já não ocasiona redução na tensão superficial. Para as soluções de DDA e OLS, a CMC foi atingida na concentração de aproximadamente $200 \mathrm{mg} / \mathrm{L}$, conforme observado na Figura 1.

\subsection{Potencial Zeta}

As Figuras 2 e 3 apresentam a variação do potencial zeta da hematita e quartzo quando em solução com os coletores oleato de sódio e dodecilamina, respectivamente.

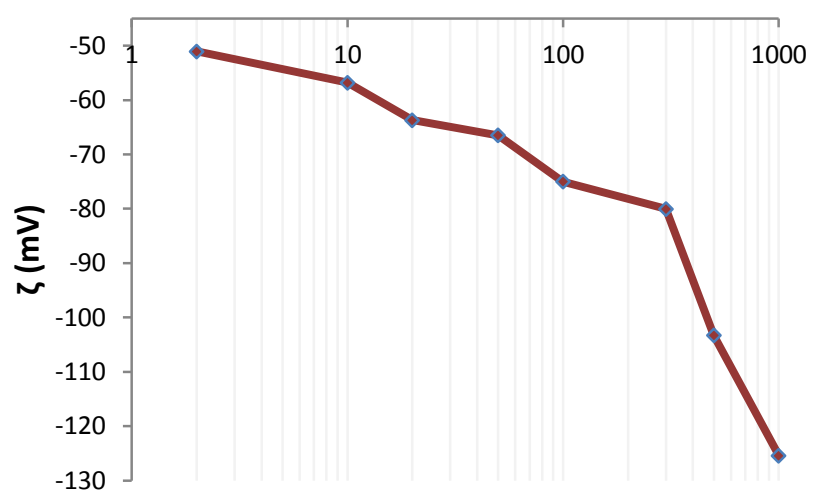

Concentração de OLS [mg/L]

Figura 2.Potencial Zeta do quartzo, em função da concentração da solução de OLS, em pH 8.

Evidencia-se pela Figura 2 que a adsorção química de oleato de sódio na superfície da hematita aumenta com a concentração de coletor, pois os valores de potencial zeta do mineral ficam cada vez mais negativos, com o aumento da concentração de oleato

\footnotetext{
* Contribuição técnica ao 69ำ Congresso Anual da ABM - Internacional e ao 14ํㅡㄹ ENEMET - Encontro Nacional de Estudantes de Engenharia Metalúrgica, de Materiais e de Minas,21 a 25 de julho de 2014, São Paulo, SP, Brasil.
} 


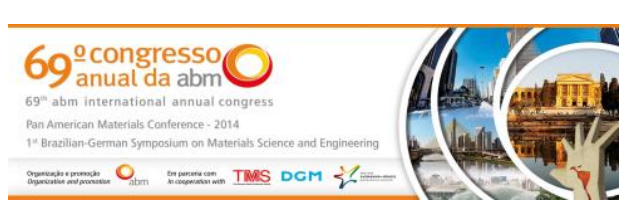

aumento da tensão superficial da solução residual, que passou de $28,6 \mathrm{mN} / \mathrm{m}$ (solução pura) para $36,0 \mathrm{mN} / \mathrm{m}$ (solução $+10 \mathrm{~g}$ de mineral). $\mathrm{O}$ valor de tensão superficial correspondente à zero de massa equivale à solução de oleato de sódio sem hematita. Conclusão semelhante pode ser estabelecida para a adsorção de dodecilamina no quartzo, conforme ilustrado na Figura 5.

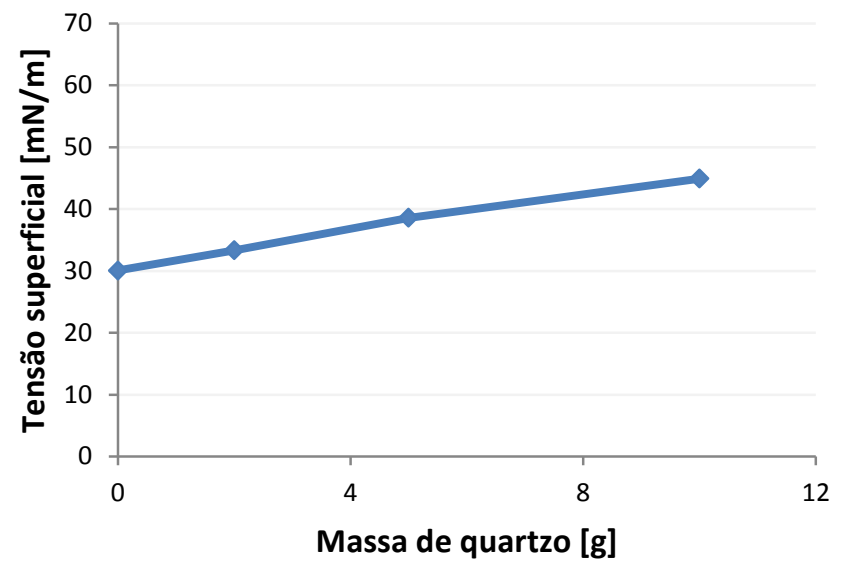

Figura 5.Tensão Superficial da solução de DDA, $200 \mathrm{mg} / \mathrm{L}$, em função da massa mineral quartzo, em $\mathrm{pH} 8$.

Nesse caso a adsorção mostrou-se mais acentuada em comparação com o ensaio utilizando hematita e oleato de sódio, cujos valores de tensão superficial passaram de $30,1 \mathrm{mN} / \mathrm{m}$ (solução pura) para $44,9 \mathrm{mN} / \mathrm{m}$ (solução + $10 \mathrm{~g}$ de mineral). O valor de tensão superficial correspondente a zero de massa equivale à solução de dodecilamina sem o mineral quartzo.

\subsubsection{Influência do tempo de adsorção}

Com o estudo cinético proposto gerou-se a curva apresentada nas Figuras 6 e 7. A curva mostra-se de acordo com que se esperava no que tange ao aumento do tempo de permanência do coletor com o mineral. Um maior tempo de exposição da massa mineral com o coletor levou a uma maior adsorção.

A Figura 6 apresenta o efeito do tempo de adsorção sobre a tensão superficial da solução de oleato de sódio residual. Analisando o comportamento da curva, observase o nítido aumento da tensão superficial da solução, sobretudo para maiores tempos de agitação da solução com o mineral, o que propicia maior adsorção e consequente aumento da tensão superficial.

\footnotetext{
* Contribuição técnica ao $69^{\circ}$ Congresso Anual da ABM - Internacional e ao 14ํㅡㄹ ENEMET - Encontro Nacional de Estudantes de Engenharia Metalúrgica, de Materiais e de Minas,21 a 25 de julho de 2014, São Paulo, SP, Brasil.
} 

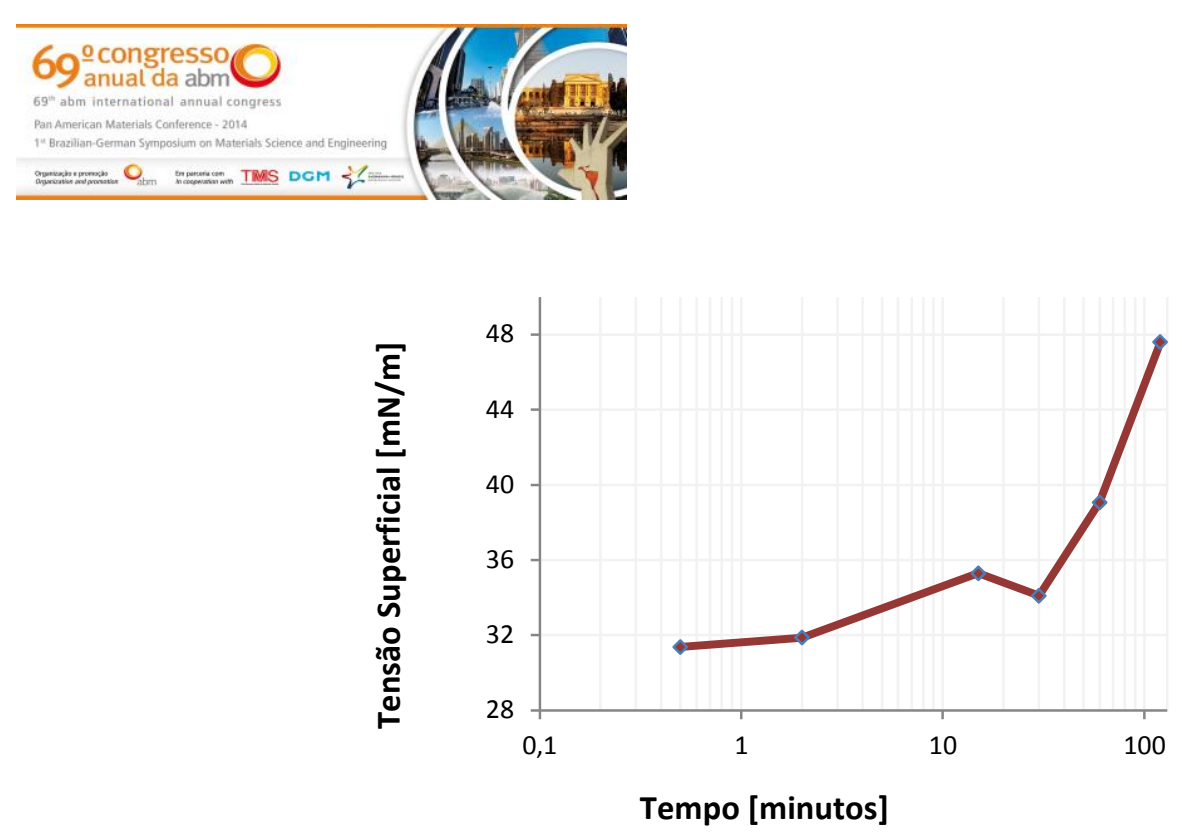

Figura 6.Tensão Superficial da solução de oleato de sódio [200mg/L] em função do tempo de adsorção, em pH8.

A Figura 7 mostra a variação da tensão superficial em função do tempo disponível para adsorção, utilizando como reagente a solução amina.

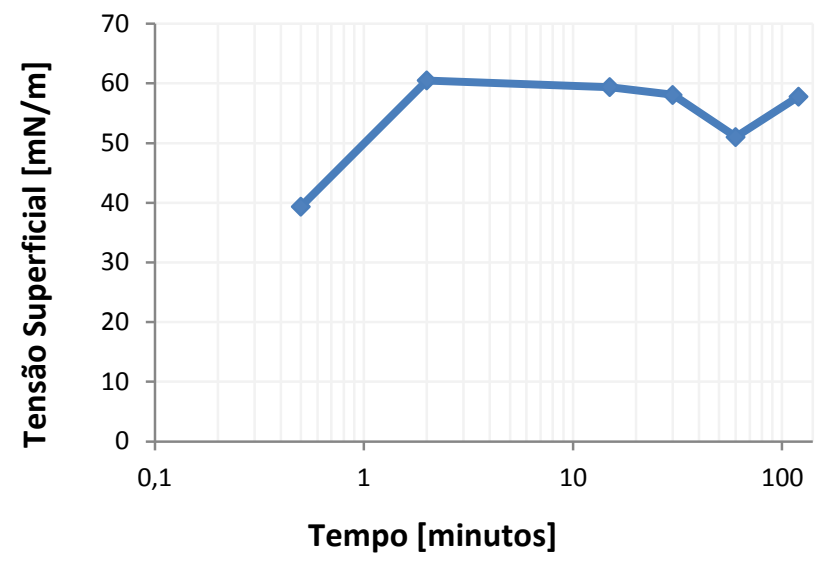

Figura 7.Tensão Superficial da solução de dodecilamina [200mg/L] em função do tempo de adsorção, em pH 8.

Para maiores tempos de adsorção, espera-se um aumento na tensão superficial. Esse comportamento ocorreu nos tempos iniciais, porém para tempos maiores houve redução na tensão superficial, seguido de um novo aumento. Esse comportamento foi de certa forma anômalo. Mesmo assim pode-se inferir que a cinética de adsorção é extremamente rápida, ocorrendo adsorção nos primeiros instantes de contato entre a solução contendo o coletor e o mineral quartzo.

\section{CONCLUSÃO}

Foi determinada a concentração micelar crítica para soluções de oleato de sódio e dodecilamina, parâmetro muito importante, pois a partir deste ponto o surfactante deixa de migrar para a interface formando micelas no interior da solução, mantendo o valor de tensão superficial constante.

Como esperado, as medidas de potencial zeta mostraram que houve adsorção, inclusive levando a reversão do potencial no caso do quartzo. No caso da hematita a grande, e progressiva, queda do potencial zeta sugere que em grandes concentrações ocorre adsorção química.

* Contribuição técnica ao 69ำ Congresso Anual da ABM - Internacional e ao 14ํㅡㄹ ENEMET - Encontro Nacional de Estudantes de Engenharia Metalúrgica, de Materiais e de Minas,21 a 25 de julho de 2014, São Paulo, SP, Brasil. 


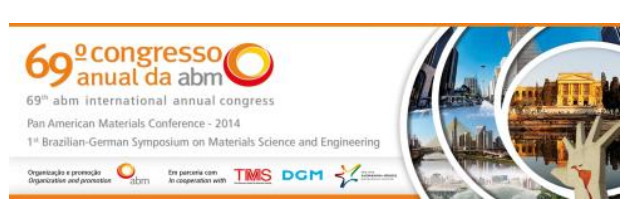

Nos testes de adsorção, realizados alterando-se a massa e o tempo de adsorção, verificou-se que ambos influenciam a adsorção. Quanto mais massa mineral disponível no sistema, maior a quantidade de coletor abstraída. E quanto maior o tempo de exposição da massa mineral com a solução de coletor, maior adsorção. Os resultados indicam que cinética de adsorção DDA sobre quartzo é mais rápida, uma vez que a adsorção ocorreu, preferencialmente, nos primeiros instantes de contato entre a solução contendo o coletor e o mineral quartzo.

\section{Agradecimentos}

Os autores agradecem à Fundação Gorceix, Capes, Fapemig e CNPq pelo apoio financeiro e concessão das bolsas.

\section{REFERÊNCIAS}

1 Neto EF. Flotação. Tecnologia Mineral. Universidade Federal do Pará. 2012.

2 Oko MU. Adsorption of Fatty Acid Soaps on Hematite. McGill University. Montreal, Canada. Set, 1965.

3 Kursun $\mathrm{H}$, Ates A. Adsorption and column flotation studies on talc using anionic and cationic collectors. Korean J. Chem. Eng. 2010;mar.:1922-1927.

4 Nascimento DR. Flotação Aniônica de Minério de Ferro. Ouro Preto: PPGEM, UFOP; 2010.

5 Lopes GM, Lima RMF. Flotação Direta de Minério de Ferro com Oletato de Sódio. REM. 2009;set.:323-329.

6 Kulkarni RD, Somasundaran P. Flotation Chemistry of Hematite/Oleate System. Colloids and Surface. 1980;mar.:387-405.

7 Shibata J, Fuerstenau DW. Flocculation and Flotation characteristics of Fine Hematite with Sodium Oleate. International Journal of Mineral Processing. 2003;jun.:25-32.

8 Viana PRM, Araújo AC, Peres AEC, Valadão GES. Adsorção de misturas de coletores em silicatos. REM: R. Esc. Minas. 2006;59(4):421-425.

9 Barbosa JLO, Baltar CAM. Influência do tamanho da partícula na flotação com amina. Holos; 2012.

10 Baltar CAM, Cunha ASF, Araújo JMM. Estudo das condições para dessorção e reutilização de coletor na flotação de quartzo. In: Anais do XIX Encontro Nacional de Tratamento de Minérios e Metalurgia Extrativa. 2002; Recife, Brasil. p.241-246.

11 Quirino L, Lima RMF. Efeito da adsorção de amina no potencial zeta da hematita e do quartzo. REM. 2003;mar.:45-49.

* Contribuição técnica ao $69^{\circ}$ Congresso Anual da ABM - Internacional e ao 14ํㅡㄹ ENEMET - Encontro Nacional de Estudantes de Engenharia Metalúrgica, de Materiais e de Minas,21 a 25 de julho de 2014, São Paulo, SP, Brasil. 